\title{
The Endovascular Armamentarium to Save the Dying Brain: Prospects and Challenges
}

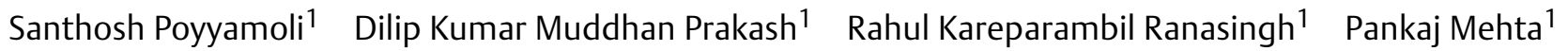 \\ Mathew Cherian ${ }^{1}$ \\ ${ }^{1}$ Department of Radiology, Kovai Medical Centre and Hospital, \\ Coimbatore, Tamil Nadu, India \\ Address for correspondence Mathew Cherian, MD, PDCC, \\ Department of Radiology, Kovai Medical Centre and Hospital, P.B. No. \\ J Clin Interv Radiol ISVIR 2017;1:32-39. \\ 3209, Avanashi Road, Coimbatore 641014, Tamil Nadu, India \\ (e-mail: dr.mathewcherian@gmail.com).
}

\begin{abstract}
Purpose Acute ischemic stroke was not long ago the territory of the neurologist, where the endovascular surgeon had only started baby steps. The scenario has completely changed post-2015 and now mechanical thrombectomy holds centerstage of the treatment guidelines. The two basic techniques for clot retrieval are the stent retriever thrombectomy and a direct aspiration first-pass technique. Use of one device as an adjunct to the other also has a passing mention in the trials. Tandem occlusions pose issues in accessing the target vessel for clot retrieval, and emergency stenting of the carotid pathway opens further avenues in stroke care.

Cases The authors present four cases of acute ischemic stroke, three of them involving the anterior circulation and one involving the basilar artery. All the cases underwent endovascular therapy, albeit with different techniques. Through the cases, they present the current technology, and its implementation and challenges faced by the interventional radiologist.

Conclusion The availability of a diverse technical repertoire helps in tackling specific

\section{Keywords}

- acute ischemic stroke

- endovascular therapy scenarios facing the stroke interventionist. The levels of evidence vary for the different weapons at hand, but it is not long before endovascular intervention becomes the panacea for stroke.
\end{abstract}

\section{Introduction}

Acute ischemic stroke (AIS) has classically been the neurologist's turf. Intravenous thrombolysis had stood the test of time, overpowering the weapons of the endovascular surgeon, with a pile of randomized controlled trials to prove its might. Endovascular therapy has come a long way from the initial days of intra-arterial thrombolysis. Modern day mechanical thrombectomy (MT) devices have made endovascular stroke therapy technically sound and a lot more efficacious.

The year 2015, however, has seen a turnaround, and will go down as a Tsunami in the history of stroke care. The benefit of MT in AIS due to emergent large vessel occlusion (ELVO) has been strongly validated in five landmark multicenter, prospective, randomized, blinded end-point clinical trials, namely the MR CLEAN, ESCAPE, EXTEND IA, SWIFT PRIME, and REVASCAT. ${ }^{1}$ The clear mandate has paved way for widespread adaptation of the technique in treating eligible acute stroke patients.

Although all these trials were based on the stent retriever thrombectomy devices, a direct aspiration first-pass technique (ADAPT) based on the principle of thromboaspiration has recently gained increasing acceptance as a cheaper and technically less challenging alternative. ${ }^{2}$

Patients who have AIS due to ELVO, and a concomitant ipsilateral high-grade internal carotid artery (ICA) stenosis or occlusion, present a greater clinical challenge. The optimum therapy in this subset of patients is still under scrutiny.

Different techniques and thoughts prevail in the realm of endovascular stroke therapy. The choice and the therapeutic result of each technique is and has been the scope of research in numerous randomized and nonrandomized trials across the globe.
DOI https://doi.org/ 10.1055/s-0036-1597909. ISSN $0000-0000$
Copyright (c) 2017 by Indian Society of Vascular and Interventional Radiology

License terms

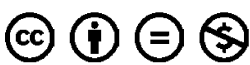




\section{Cases}

\section{Case 1}

A 42-year-old male was wheeled into the emergency room with right hemiplegia, facial palsy, and aphasia for $4 \frac{1}{2}$ hours. The National Institutes of Health Stroke Scale (NIHSS) score was 18. Alberta Stroke Program Early Computed Tomography (CT) score (ASPECTS) was 7, with a dense middle cerebral artery (MCA) sign on the left side. Magnetic resonance imaging (MRI) showed gross diffusion-perfusion mismatch with a block in the left M1 segment. Recombinant tissue plasminogen activator (rtPA) was withheld as the patient had crossed the window period. The patient was immediately shifted to the angiography suite where cerebral angiogram revealed a left M1 occlusion (-Fig. 1).
An 8F Merci (Stryker) balloon guide catheter was positioned in the left cervical ICA. Through Merci balloon catheter, Trevo Pro 18 (Stryker) microcatheter and Traxcess (MicroVention) 0.014 " microwire combination was navigated into the left MCA and positioned across the thrombus. Wire was removed and a Trevo XP ProVue $4 \times 20 \mathrm{~mm}$ stentriever (Stryker) was passed and deployed for 3 minutes. Balloon of Merci guide catheter was inflated and the stentriever was retrieved along with microcatheter, with simultaneous vigorous aspiration of the guiding catheter lumen using $50-\mathrm{mL}$ syringe. Check angiogram showed complete opening of the occlusion with thrombolysis in cerebral infarction (TICI) 3 distal perfusion.

Door-to-puncture time was 40 minutes. At 24 hours, the patient had an NIHSS score of 2. A follow-up CT showed a small left insular infarct and no hemorrhage. Modified Rankin Scale (mRS) was 1 on discharge. The mRS was 0 at 3-month follow-up.

A
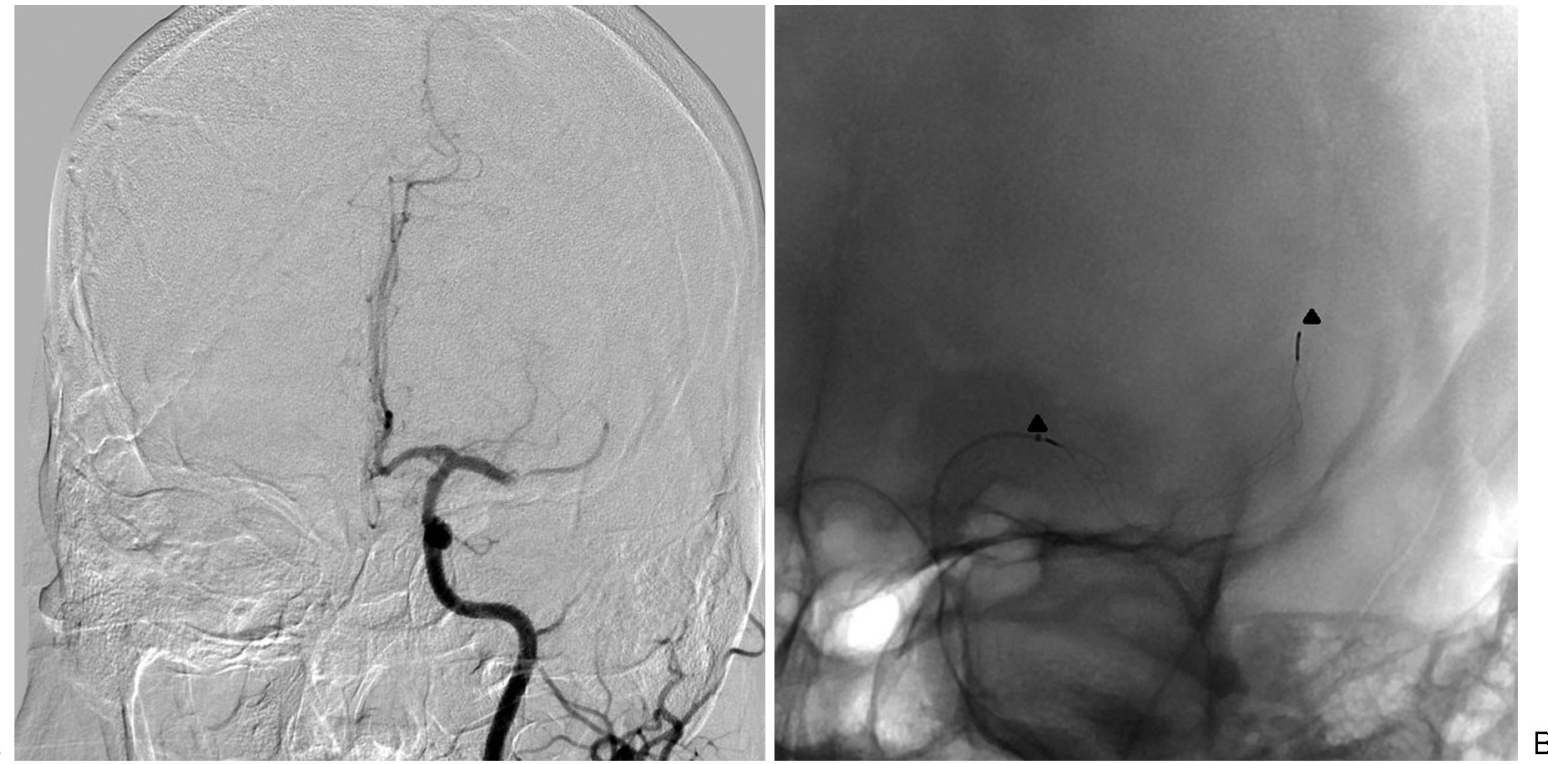

\section{B}
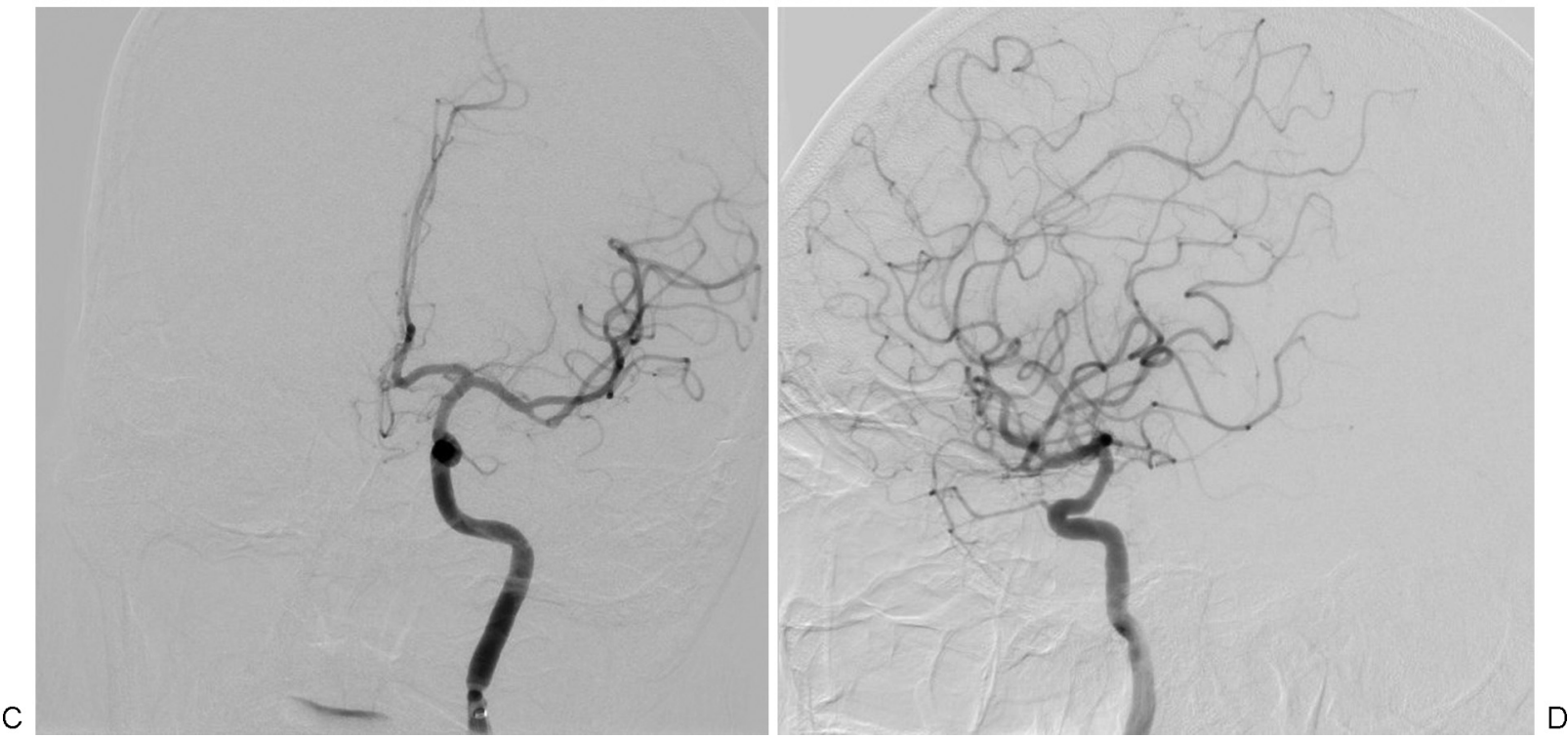

Fig. 1 (A) Left carotid angiogram shows M1 occlusion. (B) Stent retriever deployed across the occluded segment (arrowheads). (C) Anteroposterior projection shows complete recanalization of $\mathrm{M} 1$ segment. (D) Lateral projection shows filling of all cortical middle cerebral artery branches. 


\section{Case 2}

A 43-year-old male presented to the emergency room with right hemiplegia, facial palsy, and dysarthria for $2 \frac{1}{2}$ hours. The NIHSS score was 13 . ASPECTS was 10. MRI showed small hyperacute infarcts in the left side of superior pons and gross diffusion-perfusion mismatch and a basilar artery block in the left cerebellar hemisphere. rtPA was initiated and the patient was immediately shifted to the angiography suite. Left vertebral angiogram revealed occlusion of the intracranial vertebral and basilar arteries (-Fig. 2).

A 6 F Neuron (Penumbra) guiding catheter was positioned in the distal V2 segment of the left vertebral artery. A biaxial system of Headway 27 (MicroVention) and 5MAX (Penumbra) catheter was navigated over a Traxcess EX wire up to the basilar artery occlusion site. Suction thrombectomy was performed. Postprocedure check angiogram revealed com- plete recanalization of the basilar artery with TICI 3 distal perfusion.

Door-to-puncture time was 30 minutes. At 24 hours, the patient had an NIHSS score of 3 . A follow-up CT showed small pontine and cerebellar infarcts and no bleed. The mRS was 1 at discharge and 0 at 3-month follow-up.

\section{Case 3}

A 40-year-old female presented with left hemiplegia, facial palsy, dysarthria, and hemineglect for 2 hours. The NIHSS score at presentation was 12 . ASPECTS was 10. MRI showed multiple hyperacute infarcts in the entire right MCA and a diffusion-weighted imaging ASPECTS of 3. rtPA was initiated and the patient was immediately wheeled into the neurovascular catheterization laboratory. Right carotid angiogram revealed occlusion of the right M1 segment (-Fig. 3).
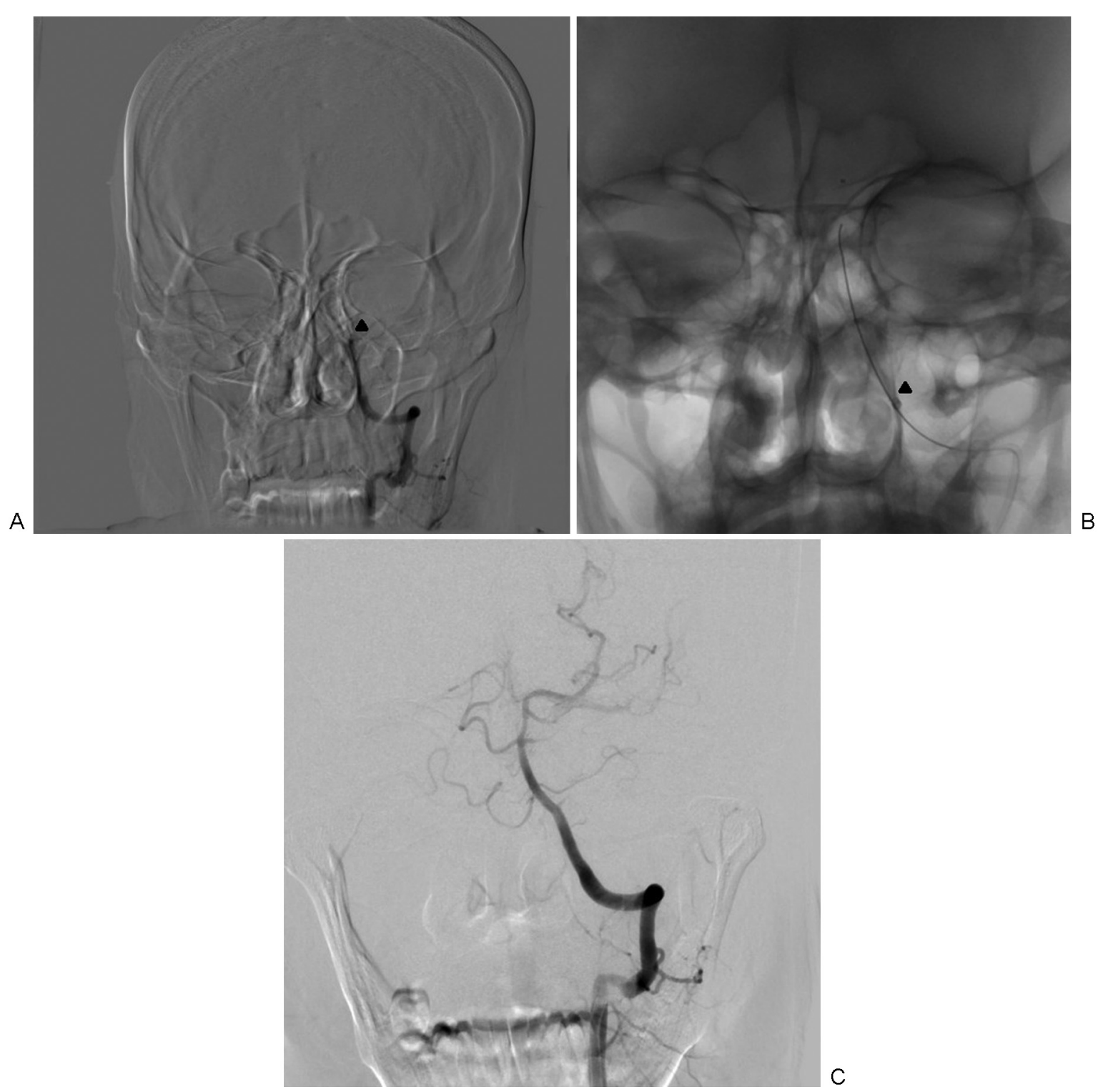

Fig. 2 (A) Left vertebral angiogram shows occlusion of left V4 segment and basilar artery (arrowhead). (B) Penumbra 5MAX catheter at the proximal end of the occlusion (arrowhead shows the radiopaque marker at the catheter tip). (C) Postsuction angiogram shows complete recanalization of the basilar artery. 


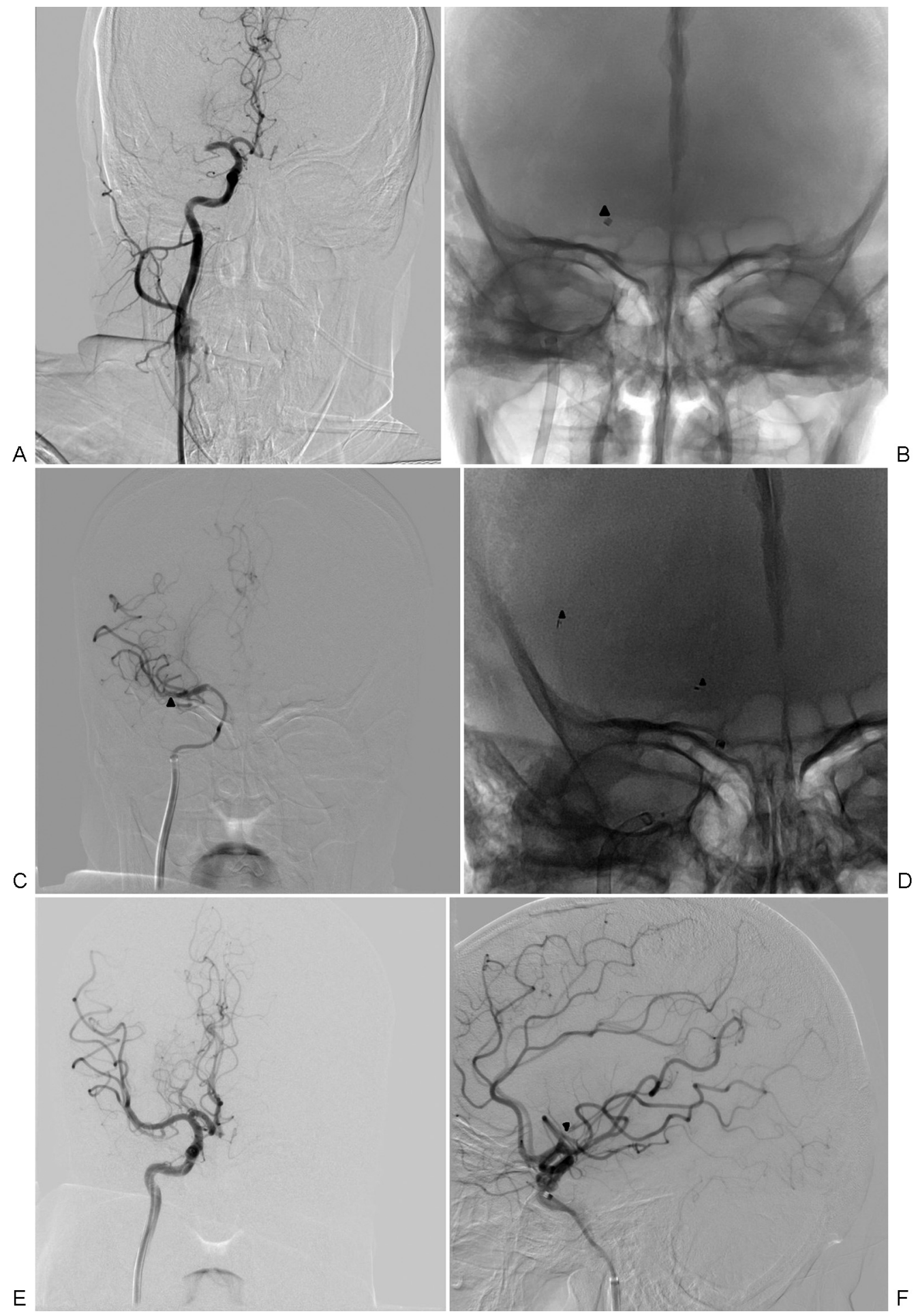

Fig. 3 (A) Right carotid angiogram shows M1 occlusion. (B) Penumbra 5MAX catheter at the proximal end of the occlusion (arrowhead shows the radiopaque marker at the catheter tip). (C) Postsuction angiogram shows persistent filling defect in the M1 segment (arrowhead). (D) Stent retriever deployed across the diseased segment (arrowheads represent end markers). (E) Postsuction angiogram; Anteroposterior projection shows complete recanalization of M1 segment. (F) Lateral projection shows persistent occlusion of the frontal branch (arrowhead) with filling of rest of the middle cerebral artery cortical branches.

An 8 F Neuron max guide catheter/sheath (Penumbra) was positioned in the petrous segment of the right ICA. A biaxial system of Penumbra 5MAX catheter, Headway 21, and Traxcess wire was navigated up to the site of the occlusion and suction thrombectomy was performed. Immediate postsuction check angiogram showed partial recanalization of the diseased segment with filling of the inferior division of right MCA. Furthermore, a Penumbra 3MAX catheter was navigated through the SMAX to the site of the superior division block. Suction thrombectomy was repeated, with 
check angiogram showing residual short segment occlusion of the superior division (M2). Further clearance of the thrombus load was achieved with use of a $6 \times 30 \mathrm{~mm}$ Preset (Phoenix) stent retriever and repeat suction with 3MAX catheter. Postprocedure check angiogram showed near complete recanalization of the right MCA with TICI 2B distal perfusion.

Door-to-puncture time was 45 minutes. At 24 hours, the patient had an NIHSS score of 8. A follow-up CT showed multiple scattered infarcts in the right MCA territory with no bleed. After a short course of rehabilitation, the NIHSS score was 5 , and mRS was 3 at discharge and 2 at 3-month follow-up.

\section{Case 4}

A 55-year-old male was rushed to the emergency room with left hemiplegia, facial palsy, hemineglect, and dysarthria for 4 hours. The NIHSS score was 16 . ASPECTS was 8. MRI showed gross diffusion-perfusion mismatch and a block in the right ICA from origin. rtPA was started and the patient was shifted to the angiography suite. Cerebral angiogram revealed occlusion of the right ICA from origin and a tandem occlusion of the right M1 segment, which was apparent in the left carotid angiogram (-Fig. 4).

An 8F Neuron Max guide catheter/sheath was positioned in the right common carotid artery. Right ICA occlusion was crossed using Choice PT 0.014" coronary wire (Boston Scientific) and dilated with Hiryu balloon (Terumo). Then the lesion was stented using 6 to $9 \mathrm{~mm} \times 30 \mathrm{~mm}$ Cristallo Ideale (Medtronic) stent. Poststenting angiogram showed complete recanalization of the diseased segment with normal cervical ICA. Right M1 occlusion was also apparent. Then a biaxial system of Penumbra 5MAX and Headway 27 microcatheter was navigated over 0.014 Traxcess guidewire to the proximal end of M1 occlusion, and suction thrombectomy was performed. Postsuction angiogram showed clearance of M1 occlusion with occlusion of frontal and parietal branches of M2 MCA, which was treated by suction thrombectomy through Penumbra Ace 3MAX catheter. Postprocedure angiogram showed persistent occlusion of the frontal branch with TICI 3 perfusion in rest of the right MCA branches. Right A1 occlusion was noted. However, there was good A2 filling through anterior communicating artery on left carotid angiogram.

Door-to-puncture time was 30 minutes. At 24 hours, the patient had an NIHSS score of 5. A follow-up CT showed small right insular and basal ganglia infarct and no bleed. The NIHSS score was 3, and mRS was 2 at discharge and 1 at 3-month follow-up.

\section{Discussion}

These cases demonstrate the wide variety of therapeutic machinery at the disposal of the interventional radiologist when summoned to treat an acute stroke patient. The technique and the equipment used to achieve timely and successful revascularization varied in each case.

In all the cases, the neuroimaging part consisted of an initial noncontrast CT (NCCT) brain in a scanner located at the emergency department where prompt decision on thrombol- ysis was taken. This process speeds up our door-to-needle time for thrombolysis. The CT was followed up with an MRI only in patients who were otherwise eligible for thrombectomy. In our center, MRI was preferred over a CT angiography of the brain for three reasons. First, CT angiography is a first-pass study, and most patients with ELVO may not sufficiently cooperate to obtain an optimal image quality, whereas sequences in a noncontrast MRI may be repeated/ optimized in case of patient movement. Second, the MR scanner is available $24 \times 7$ on an emergent basis for code stroke and is located very close to the neurovascular catheterization laboratory. Third, the MRI stroke protocol in our institute is optimized to obtain vascular imaging and noncontrast perfusion (arterial spin labeling) in a short time of 13 minutes. Overall, the "NCCT-followed-byMRI" pathway has helped us in optimal decision-making and achieving acceptable door-to-puncture times.

The first case showcased the standard and approved process for a MT, namely the stent retriever technique., ${ }^{3,4}$ This technique belonged to the second generation of the MT devices. ${ }^{5}$ It is the dominant technique used in all the five randomized control trials, which led to the 2015 update of the American Heart Association and American Stroke Association stroke treatment guidelines. Stent retrievers in the setting of AIS work by a combination of two mechanisms: (1) the deployment of the stent retriever within the clot immediately restores blood flow and (2) the mesh of stent retriever embedded within the clot serves to catch and retrieve the clot. Various stent retriever devices are available in the market (Solitaire FR, Ev3; Trevo; Revive, Codman Endovascular; Preset). An $8 \mathrm{~F}$ guide catheter is placed into the target artery using transfemoral access. A balloon guide catheter is recommended by many groups to prevent distal thrombus migration and to enhance aspiration during thrombectomy. The thrombus is crossed with a microcatheter with optional use of an intermediary catheter for enhanced support. Angiographic runs should be performed through the microcatheter to document the proper position of the microcatheter tip distal to the thrombus and to estimate the length of the clot. The stent retriever is subsequently deployed for a variable period of time, depending on the vendor specifications, covering the entire length of the occlusion. The device is slowly retrieved together with the microcatheter under continuous aspiration through the guide catheter or the intermediate catheter. In case a proximal balloon catheter is used, the balloon is temporarily inflated for the cessation of antegrade flow. The process can be repeated several times to achieve complete clot removal. ${ }^{6}$ The stent retrievers are not without drawbacks though, such as anterior cerebral artery embolism (when treating MCA or ICA occlusions), endothelial damage, and challenges associated with tortuous aortic arches, cervical, or intracranial arteries. ${ }^{5}$

The second case demonstrates the use of ADAPT technique using the Penumbra suction devices. Thromboaspiration technique belonged to the first generation of MT devices; Penumbra clot aspiration system was approved by FDA in as early as 2008 for the treatment of AIS. However, it is not until recently that there is a widespread interest in this less traumatic and faster technique. Thanks to the new 


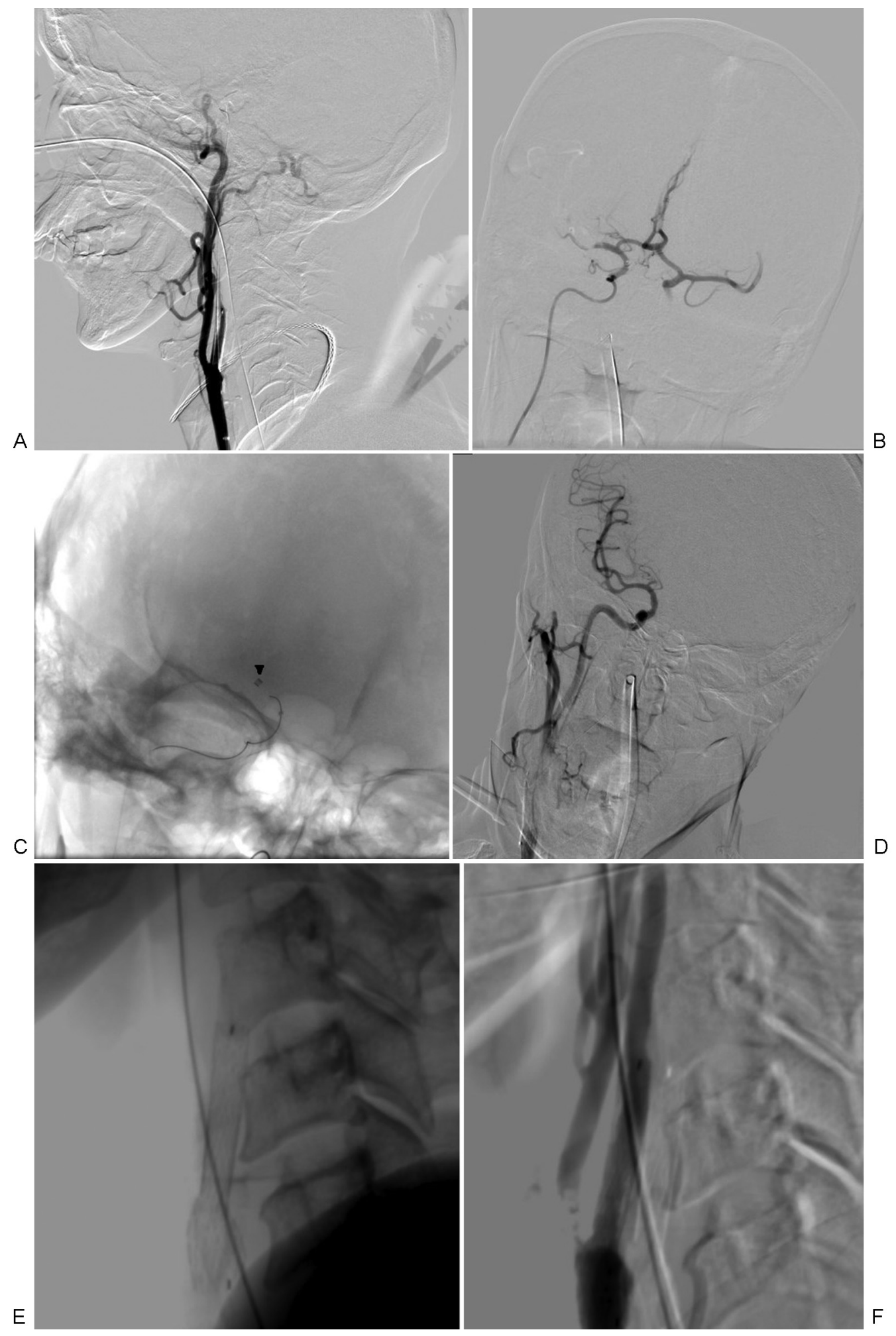

Fig. 4 (A) Right carotid angiogram shows proximal occlusion of the internal carotid artery (ICA). (B) Microcatheter injection from the intracranial ICA showed M1 occlusion. (C) Penumbra 5MAX catheter at the proximal end of the occlusion (arrowhead shows the radiopaque marker at the catheter tip). (D) Postsuction angiogram shows complete recanalization of the M1 segment. (E) Lateral projection of the neck shows the ICA stent. (F) Right carotid angiogram confirms stent patency.

developments in the suction catheter technology allowing for large bore and flexible ones that reliably navigate the twisted and tortuous arteries and those that can be employed in larger vessels like the ICA, there is renewed interest in this technique. The technique involves a guide catheter to be placed in the distal most permissible large vessel. An aspiration catheter of suitable caliber is advanced up to the proximal end of the thrombus, coaxially over a microwire and a 
microcatheter; aspiration is applied either manually with a 20 - or $50-\mathrm{mL}$ syringe or through a Penumbra aspiration pump, depending on the availability and experience. As soon as cessation of flow is noted, the catheter is slowly advanced to ensure solid engagement with the clot. Suction is continued for approximately 60 to 90 seconds. When no further flow is noticed, the catheter is removed. The number of attempts and the need for an adjunct technique are at the discretion of the interventionist. Several nonrandomized studies have documented lower procedure times and cost with comparable recanalization rates and final outcomes as compared with stent retriever thrombectomy ${ }^{2}$; albeit the lack of foolproof evidence from large randomized trials, several centers have moved on with the "starting simple" philosophy.

The third case stands as a testimony to the proverbial "unity in diversity" tagline. The two contrasting and competing techniques came together to revascularize the starving brain. Multiple attempts at ADAPT technique failed to produce a sustained recanalization, requiring the stent retriever system to come to the rescue. Two recent single-center retrospective studies stressed the importance of using stent retriever technology as an adjunct if primary thromboaspiration fails. They documented increased recanalization rates (95.3 and 87.5\%) with a combination of ADAPT and stent retriever thrombectomy than with ADAPT as a primary standalone means ( 83.3 and $43 \%$, respectively). ${ }^{7,8}$ The current generation stent retrievers can be navigated through the Penumbra 5MAX suction catheter used for the ADAPT technique, thus adding this rewarding combination to the portfolio of endovascular therapy for AIS.

The fourth case exemplifies the complex situation of a tandem occlusion where synchronous blocks of the MCA and the ipsilateral ICA pose a greater challenge in revascularization of the ischemic brain. In $\leq 20 \%$ patients with AIS, the intracranial occlusion is complicated by an additional highgrade carotid stenosis or, in the worst case, an occlusion, which further threatens endovascular access to the target vessel and delays the recanalization. ${ }^{9}$ These patients fare poorly with intravenous thrombolysis alone and at the same time pose a significant challenge to the endovascular surgeon, limiting his speed. The available literature, albeit lacking level I evidence, overwhelmingly recommends emergency cervical ICA stenting along with MT in such a scenario, either antegrade or retrograde. ${ }^{9}$ The technique employed in the index case was the antegrade approach: initial angioplasty and stenting of the carotid disease followed by MT, in this instance, thromboaspiration, of the target vessel, to achieve complete reperfusion of the ischemic territory.

In a developing country like ours, though an entire spectrum of devices is available to the neurointerventionist, the end-user cost becomes an important limiting factor in choice of the appropriate technique and equipment. In our center, we start our thrombectomy exercise with ADAPT technique, which we find to be efficacious on most occasions in isolation. Rescue therapy with a stent retriever is not attempted unless a partial success was achieved with the ADAPT technique. We, in our experience, find stent retriever less effective when there is primary failure of the ADAPT technique.

There are a slew of randomized controlled trials underway to compare the ADAPT and stent retriever techniques, and the results, either for or against one technique, will change the landscape further. If the evolution of stroke therapy is closely followed, we can correlate the breathtaking emergence of MT to the improved device design and the resultant reduction in postthrombectomy brain injury secondary to endothelial damage. This is probably reflected in the earlier clinical trials involving first-generation MT devices that could not demonstrate their superiority over intravenous thrombolysis. Future models will be designed to reduce the endothelial footprint by evolving the device design and optimizing the device size relative to the vasculature.

\section{Conclusion}

Endovascular therapy for AIS has come a long way from the days of intra-arterial thrombolysis to the modern secondgeneration MT devices. The year 2015 witnessed a tectonic shift in the ground of acute stroke care with MT being at the epicenter. The devices and techniques to achieve clot retrieval, although diverse in style and speed, often find their place, either alone or together, in the able hands of the neurointerventionist. The stent retriever is the time-tested and proven thrombectomy technique, validated across all the five landmark randomized trials of endovascular stroke therapy. The ADAPT technique, though still under the purview of randomized trials, has made great strides in many stroke centers across the globe. Using one technique as an adjunct to the other has proven to be of benefit when either of them fail to recanalize the target vessel in isolation. Finally, emergency carotid stenting added to the standard intracranial clot retrieval will be of great help to patients with tandem occlusion, who otherwise do poorly with intravenous thrombolysis alone. The evolution of MT devices is one of the most watched for spaces in the field of medical technology development. The day is not far away when endovascular therapy will become the holy grail of stroke.

\section{References}

1 Touma L, Filion KB, Sterling LH, Atallah R, Windle SB, Eisenberg MJ. Stent retrievers for the treatment of acute ischemic stroke: a systematic review and meta-analysis of randomized clinical trials. JAMA Neurol 2016;73(3):275-281

2 Romano DG, Cioni S, Vinci SL, et al. Thromboaspiration technique as first approach for endovascular treatment of acute ischemic stroke: initial experience at nine Italian stroke centers. J Neurointerv Surg 2016 (e-pub ahead of print). doi:10.1136/neurintsurg2016-012298

3 Goyal M, Demchuk AM, Menon BK, et al; ESCAPE Trial Investigators. Randomized assessment of rapid endovascular treatment of ischemic stroke. N Engl J Med 2015;372(11):1019-1030

4 English JD, Yavagal DR, Gupta R, et al. Mechanical thrombectomyready comprehensive stroke center requirements and endovascular stroke systems of care: recommendations from the Endovascular Stroke Standards Committee of the Society of Vascular 
and Interventional Neurology (SVIN). Interv Neurol 2016;4(3-4) 138-150

5 Pierot L, Soize S, Benaissa A, Wakhloo AK. Techniques for endovascular treatment of acute ischemic stroke: from intra-arterial fibrinolytics to stent-retrievers. Stroke 2015;46(3):909-914

6 Rohde S, Bösel J, Hacke W, Bendszus M. Stent retriever technology: concept, application and initial results. J Neurointerv Surg 2012; 4(6):455-458

7 Turk AS, Turner R, Spiotta A, et al. Comparison of endovascular treatment approaches for acute ischemic stroke: cost effective- ness, technical success, and clinical outcomes. J Neurointerv Surg 2015;7(9):666-670

8 Blanc R, Fahed R, Redjem H, Bartolini B, Pistocchi S, Piotin M. P031 single center experience with the ADAPT Technique for Acute Ischemic Stroke. J Neurointerv Surg 2014;6(Suppl 1): A37-A37

9 Stampfl S, Ringleb PA, Möhlenbruch M, et al. Emergency cervical internal carotid artery stenting in combination with intracranial thrombectomy in acute stroke. AJNR Am J Neuroradiol 2014; 35(4):741-746 